\title{
Evolução de condicionantes
}

\section{socioeconômicas da saúde na infância na cidade de São Paulo (1984-1996)* Secular trends in socioeconomic determinants of child health in S. Paulo city, Brazil (1984-1996)}

\section{Carlos Augusto Monteiro e Isabel Cristina Martins de Freitas ${ }^{b}$}

aD epartamento de N utrição da Faculdade de Saúde Pública da U niversidade de São Paulo. São Paulo, SP, Brasil. ' $N$ úcleo de Pesquisas Epidemiológicas em Nutrição e Saúde da U niversidade de São Paulo. São Paulo, SP, Brasil

\section{Descritores}

Indicadores de saúde".

Levantamentos epidemiológicos" Saúde infantil (saúde pública\#). Fatores socioeconômicos. Escolaridade. Renda familiar.

\section{Keywords}

Health status indicators ${ }^{\#}$. Health surveys". Child health (Public health $\left.^{\#}\right)$. Socioeconomic factors. Educational status. Family income.

\section{Resumo}

\section{Objetivo}

Descrever a evolução de condicionantes socioeconômicas da saúde na infância, com base nas informações extraídas de dois inquéritos domiciliares realizados nos anos de 1984/85 e 1995/96, na cidade de São Paulo, SP.

\section{Métodos}

Foram estudadas amostras probabilísticas da população entre zero e 59 meses de idade: 1.016 crianças em 1984/85 e 1.280 crianças em 1995/96. Os inquéritos apuraram a renda per capita mensal das famílias e o número de anos de escolaridade das mães das crianças. As rendas nominais foram deflacionadas segundo o Índice Nacional de Preços ao Consumidor e expressas em valores de outubro de 1996.

Resultados

Embora os indicadores socioeconômicos apurados no último inquérito ainda estejam muito distantes da situação ideal, a média da renda familiar dobra entre os inquéritos e a escolaridade materna aumenta em 1,5 anos. Rendas inferiores a meio saláriomínimo per capita são reduzidas à metade e virtualmente desaparece o analfabetismo materno. Ainda assim, intensifica-se no período a concentração da renda.

Conclusões

Os aumentos de renda e de escolaridade são superiores aos relatados para a população brasileira em geral, o que poderia decorrer de declínios seletivos da fecundidade nos estratos mais pobres da população de São Paulo. A influência das mudanças na renda familiar e na escolaridade materna sobre a evolução de diferentes indicadores do estado de saúde das crianças da cidade é examinada em artigos subseqüentes.

\section{Abstract}

\section{Objective}

Two consecutive household surveys undertaken in the mid-80s and mid-90s in the city of S. Paulo, Brazil, made possible to establish time trends of several child health determinants and indicators as well as to analyse the relationships among them. The study intends to report trends in socioeconomic determinants of child health.

\section{Methods}

Random samples of the population aged from zero to 59 months were studied: 1,016 children in the period 1984-85 and 1,280 children in 1995-96. Both surveys investigated the family's per capita income and the mother's years of schooling.

\footnotetext{
Correspondência para/Correspondence to: Carlos Augusto Monteiro

Av. Dr. Arnaldo, 715

01246-904 São Paulo, SP, Brasil

E-mail: carlosam@usp.br
}

*Trabalho desenvolvido no Departamento de N utrição da Faculdade de Saúde Pública da U niversidade de São Paulo e no Núcleo de Pesquisas Epidemiológicas em Nutrição e Saúde da Universidade de São Paulo e baseado em pesquisas de campo financiadas pela Financiadora de Estudos e Projetos - Finep (Convênios 41.83 .0698 .00 e 66.96.0193.00) e pela Fundação de Amparo à Pesquisa do Estado de São Paulo - Fapesp (Processos 84/2463-3 e 94/3493-5). 
Nominal incomes were deflated according to the National Consumer Prices Index and expressed as values of October 1997.

\section{Results}

From mid-80s to mid-90s average family income doubled and the proportion of low income families was reduced by 50\% while average maternal schooling increased by 1.5 years and maternal illiteracy was almost eradicated. Income concentration increased in the period.

\section{Conclusions}

Increases in income and schooling are higher than those reported for the whole population in the country, which may be attributed to selective declines in fertility among the city's poorest families. The influence that changes in family income and maternal schooling may have exerted on several child health indicators is examined in subsequent articles.

\section{INTRO DU ÇÃO}

A tendência secular das condições de saúde na infância, em qualquer população, é fortemente condicionada pela evolução do poder aquisitivo das famílias que possuem crianças e pela progressão da escolaridade dos membros destas. Do poder aquisitivo das famílias dependem, por exemplo, a disponibilidade de alimentos, a qualidade da moradia e o acesso a serviços essenciais como os de saneamento e os de assistência à saúde. Da escolaridade dos familiares, sobretudo da escolaridade materna, depende a utilização mais ou menos eficiente (do ponto de vista do cuidado infantil) da renda e dos serviços públicos que estiverem ao alcance. A escolaridade materna (e a dos demais familiares da criança) exerce influência também sobre as oportunidades de emprego e de salários e, nessa medida, condiciona o próprio poder aquisitivo das famílias.

Dois inquéritos domiciliares sucessivos, realizados em 1984/85 e 1995/96 em amostras probabilísticas da população infantil residente na cidade de São Paulo, permitem que se examine a evolução recente de um amplo conjunto de determinantes e de indicadores do estado de saúde desta população. O presente artigo aborda a evolução do poder aquisitivo das famílias das crianças, bem como a progressão do nível de escolaridade das mães destas crianças.

\section{MÉTODOS}

As informações sobre renda e escolaridade procedem de dois inquéritos domiciliares probabilísticos sobre condições de saúde na infância realizados na cidade de São Paulo em 1984/85 e 1995/96. Nos dois inquéritos foram estudadas amostras representativas da população da cidade com idades entre zero e 59 meses: 1.016 crianças no primeiro inquérito e 1.280 crianças no segundo. Os procedimentos de amostragem empregados pelos dois inquéritos partiram de cadastros domiciliares atualizados dos setores censitários do município. ${ }^{7,9}$
Os procedimentos envolvidos com a identificação do poder aquisitivo das famílias foram os mesmos nos dois inquéritos. A partir de questionários pré-testados, entrevistadores treinados obtiveram informações sobre salários e outras fontes de renda de todos os membros da unidade familiar da criança. As somatórias das rendas obtidas no mês anterior à entrevista foram então deflacionadas e expressas em reais de outubro de 1996, empregando-se, para tanto, o Índice Nacional de Preço ao Consumidor (INPC) acumulado, calculado pelo IBGE. A seguir, as rendas mensais deflacionadas foram divididas pelo número de pessoas na unidade familiar de modo a se obter a renda familiar per capita, que foi tratada como variável contínua e, também, como variável categórica. Para a constituição de quatro classes de poder aquisitivo, a renda familiar per capita foi expressa em frações do salário-mínimo vigente em outubro de 1996 ( $\mathrm{R} \$ 112,00)$ : menos de meio, entre meio e um, entre um e dois e dois ou mais salários-mínimos per capita. Em 1990, na área metropolitana de São Paulo, o atendimento das necessidades básicas das famílias apenas com alimentação implicava o dispêndio de 0,38 salários-mínimos per capita, estimando-se em cerca de 1,0 salário-mínimo per capita o dispêndio exigido para atendimento de todas as necessidades básicas. ${ }^{14}$

Os anos de escolaridade, declarados pelas mães das crianças estudadas nos dois inquéritos, foram tratados como variável contínua e como variável categórica, criando-se, nesse último caso, cinco classes: nenhum ano de escolaridade, um a três anos, quatro a sete anos (o equivalente ao antigo curso primário completo), oito a dez anos (o equivalente ao primeiro grau completo) e onze ou mais anos de escolaridade (o equivalente a pelo menos segundo grau completo).

Nos dois inquéritos, informações sobre a escolaridade materna foram obtidas para praticamente todas as famílias estudadas. Para $20,1 \%$ das famílias em 1984/85 e para 6,2\% das famílias em 1995/96 não foi possível obter informações sobre a renda de um ou mais membros da unidade familiar. Nesses casos, o 
valor da renda familiar per capita foi imputado a partir do nível de escolaridade do chefe da família. O valor da renda imputado correspondeu à renda mediana encontrada na classe de escolaridade correspondente (previamente a essa imputação, observou-se que, nos dois inquéritos, renda familiar per capita e escolaridade dos chefes nas famílias estavam altamente correlacionadas).

A evolução da renda familiar e da escolaridade materna na cidade de São Paulo foi identificada comparando-se estimativas relativas ao conjunto das crianças estudadas pelos dois inquéritos. Objetivando identificar a evolução de desigualdades sociais quanto a renda e a escolaridade, as comparações entre os dois inquéritos levaram em conta três estratos sociais "equivalentes" nas pesquisas de 1984/85 e 1995/96. Esses estratos corresponderam aos tercis da renda familiar per capita em cada inquérito, ou seja, avaliou-se a progressão ou a redução da renda familiar e da escolaridade materna no terço mais pobre, no terço intermediário e no terço mais rico das crianças da cidade.

O significado estatístico das mudanças em renda e em escolaridade, observadas entre os inquéritos, foi examinado com o emprego de análises de variância e de testes baseados na distribuição do qui-quadrado. ${ }^{5}$

\section{RESULTADOS}

Na Tabela 1, progressos substanciais e estatisticamente significantes são observados tanto no caso da renda quanto no caso da escolaridade. A média da renda familiar per capita das crianças da cidade praticamente dobra entre os inquéritos e o progresso na escolaridade materna corresponde a um acréscimo médio de 1,5 anos de educação formal.
Comparações que levam em conta a distribuição das crianças, segundo classes de renda familiar e de escolaridade materna, confirmam o progresso substancial dessas variáveis entre os inquéritos (Tabela 2). Rendas inferiores a meio salário-mínimo per capita são reduzidas em mais de 50\% (de 22,9\% para 9,3\%) e virtualmente desaparece a condição de mães sem escolaridade (de 8,2\% para 1,3\%). Ainda assim, o perfil das condições de renda e de escolaridade, que surge do inquérito mais recente, está distante do ideal. Em 1995/96, rendas inferiores a um salário-mínimo per capita, insuficientes para aquisição das necessidades básicas de uma família, conforme já mencionado, ainda caracterizavam cerca de um terço das crianças da cidade; no mesmo período, mais da metade das crianças da cidade tinham mães que não haviam sequer completado o curso de primeiro grau.

A Tabela 3 descreve a evolução das médias de renda familiar e escolaridade materna relativas a três estratos sociais da população infantil da cidade de São Paulo. No período decorrido entre os inquéritos, a renda per capita do terço mais pobre das crianças da cidade sobe 1,6 vezes, a renda do terço intermediário sobe 1,7 vezes e a renda do terço mais rico sobe 2,0 vezes. Assim, amplia-se, em termos absolutos e relativos, a diferença de renda que separa o estrato mais rico do estrato mais pobre (em termos absolutos, de 274 para 580 reais per capita; em termos relativos, de sete para nove vezes). No mesmo período, a escolaridade materna aumenta 1,6 vezes no estrato mais pobre, 1,5 vezes no estrato intermediário e 1,4 vezes no estrato mais rico, o que, nesse caso, ocasiona a diminuição discreta da diferença relativa de escolaridade entre o estrato mais rico e o mais pobre (de 2,2 vezes para 1,9 vezes), mas não a diferença absoluta (que é ampliada de 4,1 anos para 5,0 anos).

Tabela 1 - Renda familiar e escolaridade materna. Crianças entre zero e 59 meses de idade da cidade de São Paulo, SP, 1984/85 e 1995/96.

\begin{tabular}{|c|c|c|c|}
\hline Renda/Escolaridade & $\begin{array}{c}1984 / 85 \\
(n=1.016)\end{array}$ & $\begin{array}{c}1995 / 96 \\
(n=1.280)\end{array}$ & $\mathrm{p}$ \\
\hline Renda familiar per capita (R\$ de outubro/1996) & & & \\
\hline $\begin{array}{r}\bar{x} \text { e (dp) } \\
\text { Fscolaridade materna }\end{array}$ & $153,0(281,2)$ & $296,7(398,1)$ & $<0,000$ \\
\hline x e (dp) & $5,1(3,7)$ & $7,3(3,8)$ & $<0,000$ \\
\hline
\end{tabular}

Tabela 2 - Distribuição (\%) segundo estratos da renda familiar e da escolaridade materna. Crianças entre zero e 59 meses de idade da cidade de São Paulo, SP, 1984/85 e 1995/96.

\begin{tabular}{cccc}
\hline Estratos & $1984 / 85$ & $1995 / 96$ & \\
$(\mathrm{n}=1.016)$ & $(\mathrm{n}=1.280)$ & $\mathrm{p}$ \\
\hline Renda familiar per capita (salários-mínimos de outubro/1996) & & & \\
$0 \vdash 0,5$ & 22,9 & 9,3 & \\
$0,5-1,0$ & 37,9 & 24,4 & \\
$1,0-2,0$ & 24,7 & 31,2 & \\
$2,0 \vdash+$ & 14,5 & 35,1 & $<000$ \\
Escolaridade materna (anos) & 8,2 & 1,3 & \\
$1-3$ & 25,5 & 13,6 & \\
$4-7$ & 37,8 & 38,6 & \\
$8-10$ & 14,9 & 21,0 & \\
$11 \mathrm{e}+$ & 13,6 & 25,5 & \\
\hline
\end{tabular}


Tabela 3 - Renda familiar e escolaridade materna em distintos estratos sociais. Crianças entre zero e 59 meses de idade da cidade de São Paulo, SP, 1984/85 e 1995/96.

\begin{tabular}{|c|c|c|c|c|c|c|}
\hline \multirow[b]{2}{*}{ Renda/Escolaridade } & \multicolumn{2}{|c|}{$33 \%$ mais pobres } & \multicolumn{2}{|c|}{$33 \%$ intermediários } & \multicolumn{2}{|c|}{$33 \%$ mais ricos } \\
\hline & $1984 / 85$ & $1995 / 96$ & $1984 / 85$ & $1995 / 96$ & $1984 / 85$ & $1995 / 96$ \\
\hline $\begin{array}{l}\text { Renda familiar per capita (R\$ d } \\
\text { x e (dp) } \\
\text { Escolaridade materna (anos) }\end{array}$ & $\begin{array}{l}0 / 1996) \\
44,6(18,4)\end{array}$ & $71,8^{*}(28,4)$ & $95,4(14,3)$ & $163,9^{*}(34,6)$ & $318,2(440,6)$ & $652,2^{*}(527,0)$ \\
\hline$x \in(d p)$ & $3,4(2,7)$ & $5,3^{*}(2,7)$ & $4,2(2,6)$ & $6,3^{*}(2,9)$ & $7,5(4,2)$ & $10,3^{*}(3,6)$ \\
\hline
\end{tabular}

*p<0,000.

\section{ISC U SSÃO}

Os aumentos de renda e de escolaridade revelados pelos inquéritos realizados na cidade de São Paulo, não surpreendem, visto ser essa a tendência predominante apontada, no período, em todas as regiões do País, pelas Pesquisas Nacionais por Amostra de Domicílios PNADs. ${ }^{6} \mathrm{O}$ que surpreende é a magnitude dos aumentos observados em São Paulo. Por exemplo, as PNADs realizadas em 1985 e em 1995, próximas portanto aos dois inquéritos realizados em São Paulo, indicam aumento da renda per capita (também em reais de outubro de 1996) para todas as famílias do país de $\mathrm{R} \$ 246,00$ para $\mathrm{R} \$ 271,00$ e, para as famílias residentes nas áreas urbanas da Região Sudeste, de R \$ 329 para R \$ 366. Segundo os inquéritos realizados na cidade de São Paulo, a renda familiar per capita relativa às crianças menores de cinco anos dobra no período, elevando-se de $\mathrm{R} \$ 153,00$ para $\mathrm{R} \$ 296,70$.

Estimativas das PNADs de 1985 e 1995, sobre a escolaridade da população feminina das áreas urbanas da Região Sudeste, igualmente indicam melhorias mais modestas do que as observadas para a escolaridade materna na cidade de São Paulo. Por exemplo, a escolaridade média das mulheres adultas ( 25 ou mais anos de idade) residentes no Sudeste urbano eleva-se, no período, de 5,2 para 6,0 anos (contra um aumento de 5,1 para 7,3 anos no caso da escolaridade materna em São Paulo). No mesmo período, as PNADs indicam que o analfabetismo entre mulheres jovens ( 25 a 29 anos) do Sudeste urbano reduz-se de $4,8 \%$ para $3,1 \%$ (crianças de mães sem escolaridade declinam de $8,2 \%$ para 1,3\% na cidade de São Paulo).

A melhoria mais intensa dos indicadores de renda e de escolaridade evidenciada pelos inquéritos relativos à população infantil de São Paulo comporta duas possíveis explicações. A primeira seria a evolução mais favorável da economia e das oportunidades de educação na cidade de São Paulo do que nas demais áreas urbanas da Região Sudeste. A segunda possibilidade a considerar seria que a intensa melhoria da renda e da escolaridade estaria restrita à população infantil da cidade. A primeira possibilidade não encontra respaldo em qualquer evidência ou argumento. A segunda pressupõe mudanças substanciais na dinâmica demográfica da cidade de
São Paulo. Declínios da fecundidade, que alcançassem particularmente as mulheres de menor renda e de menor escolaridade da cidade, "produziriam" melhorias no perfil socioeconômico do segmento infantil da população que não teriam paralelo com a evolução da população geral da cidade. Declínios seletivos da fecundidade na cidade, particularmente intensos entre mulheres sem instrução, foram relatados na população da Grande São Paulo no período 1980-1986. ${ }^{4}$ Há também evidências de que, nos anos 90, o declínio da fecundidade na população urbana brasileira ocorreu de forma muito mais intensa nas regiões menos desenvolvidas do País (Norte e Nordeste), fenômeno explicado pelas taxas já muito baixas da fecundidade nas regiões mais desenvolvidas (Centro-Sul). ${ }^{8}$ Alterações no perfil social e demográfico das famílias com crianças pequenas, que emigram de São Paulo ou imigram para São Paulo, igualmente poderiam contribuir para gerar "defasagens" entre a evolução do perfil socioeconômico da população infantil e o da população geral da cidade, mas, nesse caso, não há evidências empíricas que dêem respaldo à hipótese.

Cabe ainda notar que o aumento da renda familiar observado entre os inquéritos realizados em São Paulo não foi acompanhado de melhorias na distribuição social do poder aquisitivo da população. Ao contrário, no período, aumentou de sete para nove vezes a diferença existente entre a renda média do terço mais rico e a do terço mais pobre da população infantil de São Paulo. No mesmo período, a diferença de escolaridade (número de anos cursados) entre as mães do terço mais rico e as do mais pobre das crianças da cidade foi ligeiramente reduzida em termos relativos, mas elevou-se em termos absolutos. Situações semelhantes quanto à distribuição da renda familiar, ou seja, manutenção ou mesmo exacerbação de distribuições extremamente concentradas, são características de todas as regiões brasileiras. ${ }^{6}$

A influência que as mudanças observadas na renda familiar e na escolaridade materna podem ter exercido sobre a evolução de diferentes indicadores do estado de saúde das crianças de São Paulo - envolvendo a nutrição pré e pós-natal, o crescimento pondoestatural e as enfermidades mais freqüentes na infância - é examinada em artigos subseqüentes..$^{1-3,10-13}$ 


\section{REFERÊNCIAS}

1. Benicio MHD'A, Monteiro CA. Tendência secular da doença diarréica na infância na cidade de São Paulo (1984-1996). Rev Saúde Pública 2000;34(6 Supl):83-90.

2. Benicio MHD'A, Cardoso MRA, Gouveia NC, Monteiro CA. Tendência secular da doença respiratória na infância na cidade de São Paulo (1984-1996). Rev Saúde Pública 2000;34(6 Supl):91-101.

3. Ferreira MU, Ferreira CS, M onteiro CA. Tendência secular das parasitoses intestinais na infância na cidade de São Paulo (1984-1996). Rev Saúde Pública 2000;34(6 Supl):73-82.

4. Fundação SEADE. A fecundidade da mulher paulista. São Paulo: Fundação SEADE; 1994. (Informe Demográfico, 25).

5. Kirkwood BT. Essentials of medical statistics. London: Blackwell; 1988. p. 234.

6. Lavinas $L, M$ agina $M$, coordenadores. Atlas regional das desigualdades: banco de dados com indicadores sócioeconômicos por U.F. e macroregiões. Rio de Janeiro: IPEA/DIPES; 1996.

7. Monteiro CA, Pino Z HP, Benicio MHD'A, Szarfarc SC. Estudo das condições de saúde das crianças do Município de São Paulo (1984/85). I. Aspectos metodológicos, características socioeconômicas e ambiente físico. Rev Saúde Pública 1986;20(6):435-45.
8. Monteiro CA, Benicio MHD'A, Freitas ICM. Melhoria em indicadores de saúde associados à pobreza no Brasil dos anos 90: descrição, causas e impacto sobre desigualdades regionais. São Paulo: NU PEN S/U SP; 1997. (A Trajetória do Desenvolvimento Social no Brasil, 1/97].

9. Monteiro CA, Silva N N, Nazário CL. A pesquisa de campo de 1995/96. In: Monteiro CA, organizador. Como e por que melhoram (ou pioram) os indicadores de saúde e nutrição na infância ? O caso da cidade de São Paulo na segunda metade do século XX. São Paulo: NUPENS/ USP; 1999. (Relatório Técnico - FAPESP, julho/1999).

10. Monteiro CA, Conde W L. A tendência secular do crescimento pós-natal na cidade de são Paulo (19741996). Rev Saúde Pública 2000;34(Supl):41-51.

11. Monteiro CA, Conde WL. Tendência secular da desnutrição e da obesidade na infância na cidade de São Paulo (1974-1996). Rev Saúde Pública 2000;34(6 Supl):52-61.

12. Monteiro CA, Benicio MHD 'A, O rtiz LP. Tendência secular do peso ao nascer na cidade de São Paulo (1976-1998). Rev Saúde Pública 2000;34(6 Supl):26-40.

13. Monteiro CA, Szarfarc SC, Mondini L. Tendência secular da anemia na infância na cidade de São Paulo (19841996). Rev Saúde Pública 2000;34(6 Supl):62-72.

14. Rocha S. Governabilidade e pobreza: o desafio dos números. Rio de Janeiro: IPEA; 1995. (Texto para Discussão, 368). 